\title{
Traduire
}

Revue française de la traduction

$233 \mid 2015$

Voyage en équipage

\section{Le sous-titrage interlinguistique : comment transférer les valeurs sémantiques des marqueurs discursifs}

\section{Rania A. Khalifa}

\section{(2) OpenEdition \\ Journals}

\section{Édition électronique}

URL : http://journals.openedition.org/traduire/774

DOI : 10.4000/traduire.774

ISSN : 2272-9992

Éditeur

Société française des traducteurs

\section{Édition imprimée}

Date de publication : 15 décembre 2015

Pagination : 96-117

ISSN : 0395-773X

\section{Référence électronique}

Rania A. Khalifa, «Le sous-titrage interlinguistique : comment transférer les valeurs sémantiques des marqueurs discursifs », Traduire [En ligne], 233 | 2015, mis en ligne le 15 décembre 2017, consulté le 19 avril 2019. URL : http://journals.openedition.org/traduire/774; DOI : 10.4000/traduire.774 


\section{Le sous-titrage interlinguistique : comment transférer les valeurs sémantiques des marqueurs discursifs}

Rania A. Khalifa

\section{Introduction}

Transfert d'un donné sonore en un donné visuel, recette conseillée pour transcender la barrière de la langue, le sous-titrage est une des multiples facettes de la traduction audiovisuelle qui gagne en importance et coûte selon les estimations dix fois moins cher que le doublage, tout en préservant l'identité originale du document audiovisuel.

Dans son article "Les sous-titres, un mal nécessaire ", Lucien Marleau distingue six fonctions des sous-titres : fonction de remplacement (remplacer l'oral par l'écrit), de communication (médiatiser indirectement et à sens unique), émotive (susciter chez le spectateur la même affectivité que le film original), d'ancrage (préciser le sens de l'image), de relais (prendre en charge des éléments d'information non inclus dans l'image) et de redondance (répéter ce que dit l'image) (Marleau, 1982 : 274).

\section{Démarche d'analyse}

Notre objectif est d'apporter aux réflexions traductologiques une nouvelle perspective relative au rapport complexe oral vs écrit. Nous essayerons de déterminer dans quelle mesure le soustitrage, qui permet le passage d'une langue (le français) à une autre (l'arabe) et d'un code (oral) à un autre (écrit), participe à la production du sens discursif et est capable de transférer la saillance des valeurs et fonctions des marqueurs discursifs.

Tout au long de notre recherche, nous analyserons la place des marqueurs discursifs dans le sous-titrage qui constitue une des trois composantes du langage télévisé : le langage sonore (en l'occurrence le français dans le cas de l'émission de TV5 que nous allons étudier), le langage non verbal et le langage visuel inséré dans le bas de l'écran. Et nous verrons quelle représentation de " ces petits mots " le sous-titrage favorise.

Nous avons choisi comme corpus une émission du magazine MOE (Maghreb Orient Express) tournée en Égypte et diffusée le 11 septembre 2011 sur TV5. Riche en marqueurs discursifs, 
l'émission est un terrain qui nous permet d'étudier une composante de la traduction audiovisuelle, de comparer le produit reçu par le sous-titreur et celui que lit le spectateur de l'émission sous-titrée, et par là de déterminer si les marqueurs discursifs ont joué le même rôle dans les deux textes.

La problématique explorée correspond aux questions suivantes : le sous-titrage contribue-t-il à une surinformation ou plutôt à une sous-information ? Le transfert du signifié linguistique vers une langue cible se fait-il forcément au détriment de la langue source ? Le passage du sens discursif du français vers l'arabe signifie-t-il obligatoirement une perte de sa valeur illocutoire, ou au contraire un ajout à cette valeur?

Nous sommes partis du postulat suivant : de par la concision du sous-titrage, certains éléments linguistiques aussi bien que non linguistiques se perdent et s'estompent, d'où un produit final sous-titré moins informatif que le texte original. Nous tenterons de voir dans quelle mesure cette hypothèse est vérifiée dans le cadre de l'étude du potentiel discursif des marqueurs.

L'émission commence avec le présentateur de l'émission (Mohamed Kaci) et quatre invités ; deux d'entre eux quittent ensuite le plateau et sont remplacés par deux autres. Durant toute l'émission, c'est le présentateur qui pose les questions aux invités, c'est lui qui dirige toute l'interaction. Et lorsqu'un invité cherche à commenter une idée évoquée par un autre, il ne le fait que lorsque le présentateur lui donne la parole. L'interaction suit donc le schéma suivant:

\section{Première réception (émission originale)}

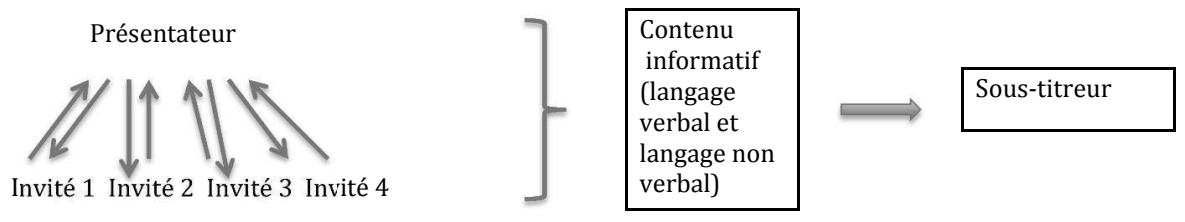

Toutefois, la première réception décrite par le schéma ci-dessus est suivie d'une deuxième réception, celle de l'émission sous-titrée.

\section{Deuxième réception (émission sous-titrée)}

Présentateur

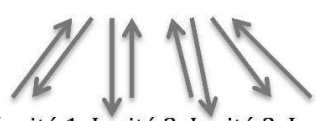

Invité 1 Invité 2 Invité 3 Invité 4

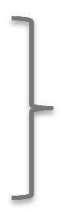

Téléspectateurs arabophones et francophones 
Nous chercherons dans les pages qui suivent à vérifier si les marqueurs discursifs propres à la langue orale gardent dans le sous-titrage leur force illocutoire et leur fonction discursive d'origine.

\section{Marqueurs discursifs et sens procédural}

Les marqueurs discursifs (MD) ont intéressé un grand nombre d'auteurs tant d'un point de vue définitoire que terminologique. "Mot de discours " (Ducrot et al. 1980), " particule énonciative " (Fernandez, 1994), " opérateur " (Anscombre et Ducrot, 1983), " organisateurs textuels " (Adam, 1990), " marqueurs de structuration de la conversation " (Auchlin, 1981), " marqueurs de reformulation paraphrastique " (Gülich et Kotchi, 1983) sont des étiquettes qui renvoient le plus souvent à une seule et même classe. Néanmoins, le terme le plus répandu demeure de loin celui de "marqueurs discursifs". Shiffrin (1987), Hansen (1998) et Fraser (1999) se sont accordés sur le fait que le rôle principal des MD était de lier les différentes parties d'un énoncé, permettant cohésion et cohérence. Ils jouent cependant un rôle au-delà du cadre de la phrase, en ce sens qu'ils sont indépendants de la valence du verbe. Ils contribuent à l'interprétation d'un discours et permettent au locuteur de se positionner par rapport à son discours. Selon Claire Blanche-Benveniste (1997), les MD sont morphologiquement invariables, ils ne contribuent pas au contenu propositionnel des énoncés et c'est pourquoi leur présence ou leur absence ne modifie pas la valeur de vérité des énoncés auxquels ils sont joints. Syntaxiquement parlant, leur absence n'entraîne pas d'agrammaticalité. De plus, ils jouent un rôle audelà du cadre de la phrase et relèvent de la macro-syntaxe du discours.

Une des caractéristiques des MD est la situation d'interlocution, puisqu'ils permettent au destinataire de se positionner par rapport à son discours ou par rapport à celui de son interlocuteur pour le bénéfice de ce dernier.

Les MD doivent être envisagés dans [le cadre] de la langue orale, où la coprésence de l'interlocuteur influence la façon dont le locuteur construit son discours. Ils apparaissent à des endroits stratégiques et ils contribuent à rendre efficaces les échanges conversationnels, ainsi qu'à aider l'interlocuteur à décoder la façon dont le locuteur conçoit le sens purement propositionnel exprimé et se positionne par rapport à celui-ci. (Dostie et Pusch, 2007 : 5)

Le tableau ci-dessous résume les caractéristiques de base des marqueurs discursifs. 


\begin{tabular}{|l|l|}
\hline \multicolumn{1}{|c|}{ Traits sémantiques et pragmatiques } & \multicolumn{1}{c|}{ Traits morphologiques } \\
\hline $\begin{array}{l}\text { - Sens procédural. } \\
\text { - Rôle dans l'optimisation de l'interaction verbale. }\end{array}$ & $\begin{array}{l}\text { - Invariables ; peuvent s'associer entre eux pour former } \\
\text { des marqueurs complexes. } \\
\text { - Indices de l'oralité du discours. }\end{array}$ \\
\hline \multicolumn{1}{|c|}{ Traits syntaxiques } & \multicolumn{1}{c|}{ Traits phonétiques } \\
\hline $\begin{array}{l}\text { - Position variable dans l'énoncé. } \\
\text { Leur suppression n'entraîne pas d'agrammaticalité de } \\
\text { lacultative. }\end{array}$ & $\begin{array}{l}\text { - Peuvent présenter une réduction phonologique. } \\
\text { - Monosyllabiques pour la plupart. } \\
\text { - Le plus souvent marqués sur le patron intonatif } \\
\text { (intonation particulière). }\end{array}$ \\
\hline
\end{tabular}

Tableau 1. Traits caractéristiques des marqueurs discursifs

Nous sommes partis du modèle sémantico-cognitif de l'énoncé original (français) pour parvenir à la dimension linguistique de l'énoncé sous-titré (en arabe).

Notre analyse s'est déroulée en deux étapes. Dans la première, nous avons recensé les MD les plus fréquents dans l'énoncé français et nous avons cherché à savoir s'ils étaient traduits dans l'énoncé arabe ou non. Les résultats de cette première étape figurent dans le tableau suivant :

\begin{tabular}{|l|c|c|c|}
\hline \multicolumn{1}{|c|}{ MD } & $\begin{array}{c}\text { Nombre d'occurrences } \\
\text { dans la version originale } \\
\text { (VO) }\end{array}$ & $\begin{array}{c}\text { Nombre d'occurrences } \\
\text { maintenues dans la ver- } \\
\text { sion sous-titrée (VST) }\end{array}$ & $\begin{array}{c}\text { Pourcentage de maintien } \\
\text { du marqueur }\end{array}$ \\
\hline Mais & 59 & 55 & $93 \%$ \\
\hline Donc & 35 & 10 & $28 \%$ \\
\hline Aussi & 28 & 13 & $46 \%$ \\
\hline Alors & 15 & 9 & $60 \%$ \\
\hline Quand même & 12 & 2 & $16 \%$ \\
\hline Toujours & 11 & 6 & $54 \%$ \\
\hline Peut-être & 11 & 5 & $45 \%$ \\
\hline Là & 13 & 5 & $38 \%$ \\
\hline Voilà & 8 & 0 & $0 \%$ \\
\hline En tout cas & 5 & 2 & $40 \%$ \\
\hline C'est-à-dire & 5 & 2 & $40 \%$ \\
\hline Je veux dire & 5 & 0 & $0 \%$ \\
\hline En revanche & 4 & 3 & $75 \%$ \\
\hline D'ailleurs & 4 & 0 & $0 \%$ \\
\hline Enfin & 4 & 1 & $25 \%$ \\
\hline Je ne sais pas & 3 & 1 & $33 \%$ \\
\hline On ne sait quoi & 1 & 1 & $100 \%$ \\
\hline Comment dire & 2 & 1 & $50 \%$ \\
\hline Vous voyez & 1 & 0 & $0 \%$ \\
\hline
\end{tabular}

Tableau 2. Marqueurs discursifs du corpus et pourcentage de reprise dans les sous-titres 
Comme le montre le tableau, le terme MD recouvre aussi pour nous ce que la littérature pragmatique s'accorde à appeler " connecteurs " et " particules ". Le décalage entre la V0 et la VST est manifeste.

Dans une deuxième étape, nous allons étudier plus en détail quelques marqueurs représentatifs. Deux critères ont déterminé leur choix :

- la disproportion entre leur apparition dans la VO et dans la VST (marqueurs représentatifs qui figurent dans l'émission française et ont complètement disparu dans l'énoncé arabe, tels que " voilà ", " je veux dire " et " vous voyez ") ;

- la variété de leur fonction (marqueurs et particules qui couvrent des champs divers tels que la progression discursive, la préservation du tour de parole et la structuration de l'interaction). C'est la raison pour laquelle nous avons retenu les marqueurs " donc " et " alors ", ainsi que des marqueurs propositionnels.

Nous avons de même adopté une approche ascendante qui cherche à déterminer la gamme fonctionnelle de certains marqueurs discursifs, selon les contextes.

\section{1. " Donc ", marqueur ou connecteur}

Le marqueur discursif " donc " constitue un marqueur de grande importance du fait qu'il peut structurer le discours à différents niveaux langagiers, dépassant de la sorte sa fonction de connecteur conséquentiel. De nombreuses études ont analysé cette particule, ses caractères sémantiques et ses fonctions discursives.

Zenone (1981) souligne cinq emplois de " donc " (de reprise, discursif, argumentatif, métadiscursif et récapitulatif), avant de les répartir en deux groupes : cotextuel (fonction argumentative) et contextuel (autres fonctions).

En 1997, M.-B. M. Hansen, dans son analyse de "donc ", a emprunté la notion de mutual manifestness (évidence mutuelle), impliquant que le locuteur et l'allocutaire conçoivent une chose de la même façon. Elle définit trois fonctions, ou propriétés syntactico-sémantiques, de "donc " : marqueur de conclusion et de conséquence, marqueur de répétition et particule modale. Dans les deux premières fonctions, l'information introduite par " donc " est connue et mutuellement manifeste pour les co-énonciateurs.

Certes, la fonction principale de " donc " connecteur est de " rendre légitime ou plausible la proposition marquée par le connecteur : l'usage de "donc" autorise le locuteur à présenter cette proposition comme vraie et comme seule conclusion possible inférable à partir des prémisses et propositions antérieures" (Bolly et Degand, $2009: 6)$.

Nous avons adopté la taxinomie établie par Catherine Bolly et Liesbeth Degand. Ces deux linguistes ont recensé cinq fonctions sémantiques et/ou pragmatiques de "donc " : marqueur 
de conséquence, de répétition à orientation conclusive, de reformulation, de transition participative et de structuration conceptuelle. À ces cinq fonctions, nous pouvons ajouter une sixième, à savoir la fonction modale : " donc " ponctuant et phatique, bien distingué au niveau intonatif, qui vise à délimiter les différentes unités participatives ou syntaxiques.

Dans notre corpus, il nous a fallu nous focaliser sur les données contextuelles pour déterminer la valeur précise de "donc". Les fonctions de ces occurrences varient entre des fonctions syntactico-sémantiques (en tant que connecteur), ou des fonctions discursives (en tant que marqueur discursif).

\begin{tabular}{|l|l|c|c|}
\hline \multicolumn{2}{|l|}{} & Fréquence & Pourcentage \\
\hline \multirow{2}{*}{ Type de lien } & Conséquence & 8 & $22,8 \%$ \\
& Récapitulation & 5 & $14,2 \%$ \\
& Reformulation + explicitation & 11 & $31,4 \%$ \\
& Transition participative & 3 & $8,5 \%$ \\
& Transition de structure conceptuelle & 3 & $8,5 \%$ \\
& Ponctuant & 5 & $14,2 \%$ \\
\hline \multicolumn{2}{|c|}{ Total } & 35 & $100 \%$ \\
\hline
\end{tabular}

Tableau 3. Différentes fonctions de " donc " dans notre corpus oral

L'analyse des différents " donc " a mis en évidence que dans notre corpus la fonction discursive de ce marqueur l'emporte sur la fonction sémantique. La fonction de répétition à orientation conclusive (récapitulation) ainsi que la reformulation, l'explicitation et les transitions sont presque trois fois plus fréquentes que la relation conséquentielle. Mais qu'en est-il dans la traduction en arabe?

Le tableau suivant montre que le sous-titreur a supprimé $82 \%$ des " donc ", $18 \%$ seulement des occurrences de la version originale étant conservés dans le sous-titrage. II s'agit pour la plupart de cas liés à la fonction sémantique plutôt qu'à la fonction discursive.

\begin{tabular}{|l|l|c|c|}
\hline \multicolumn{2}{|c|}{} & Maintien & Disparition \\
\hline Type de lien & Conséquence & 4 & 4 \\
& Récapitulation & 2 & 3 \\
& Reformulation + explicitation & 0 & 11 \\
& Transition participative & 0 & 3 \\
& Transition de structure conceptuelle & 0 & 3 \\
& Ponctuant & 0 & 5 \\
\hline \multicolumn{1}{|c|}{ Total } & 6 & 29 \\
\hline
\end{tabular}

Tableau 4. Différentes occurrences de la traduction de "donc " dans le corpus sous-titré 
Un décalage entre la réception des deux textes est par conséquent manifeste. Dans l'exemple suivant, la fonction sémantique de " donc " a disparu.

- Exemple 1 : oui + vu ce qui s'est passé en Norvège ++ donc + aujourd'hui + le terrorisme n'est pas seulement lié à l'arabe ou le musulman + ou Ben Laden(1)

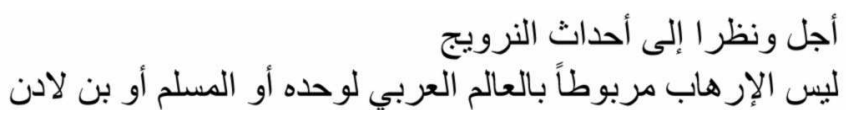

La relation conséquentielle est claire en français : I'assassinat qui a eu lieu en Norvège est un message au monde entier que le terrorisme, l'intégrisme et l'extrémisme existent partout - des étiquettes que le Nord tentait d'attribuer aux musulmans. Le sous-titreur en ayant recours à la lettre arabe ف ف (relative à la causalité) aurait pu montrer cette relation. De même, le complément de temps (aujourd'hui) a été annulé, alors que son maintien nous paraît primordial pour montrer la modification qui s'est opérée au sein de l'opinion publique internationale. Sur l'axe du temps, l'assassinat en Norvège a contribué à effacer la stigmatisation des Arabes. Le verbe arabe لم يعد (n'est plus) suffit à lui seul à transférer cette valeur sémantique sans

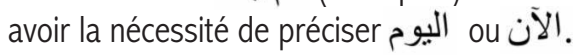

La traduction que nous proposons est la suivante :

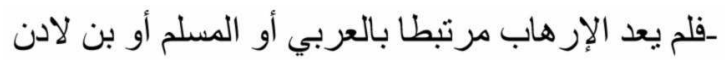

Elle a le mérite d'être plus pertinente et plus courte.

Voyons l'exemple suivant :

- Exemple 2 : les effets négatifs du 11 septembre + enfin + comme moi je ne vois pas vraiment un lien + par rapport à ce qui a été créé entre $2001++$ c'est-à-dire + c'est quelque chose d'après moi + en tant que méditerranéenne ++ une chose créée par le Nord + cela donc + je n'arrive pas à faire un rapport

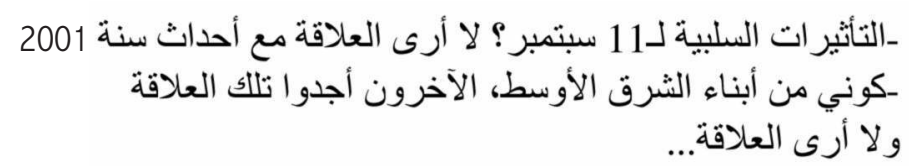

Cet exemple nous paraît assez intéressant puisqu'il comprend une cooccurrence de " donc " marqueur de conséquence et marqueur de reformulation. L'invitée est en train d'expliquer la raison pour laquelle elle ne trouve pas de lien entre les événements du 11 septembre et le printemps arabe. Son raisonnement suit un cheminement logique qui l'amène à réfuter tout rapport

(1) Les règles de transcription sont inspirées des conventions du GEDO/GARS : aucun signe de ponctuation, les majuscules sur les noms propres, + pause courte, ++ pause longue, L1 locuteur premier, L2 locuteur second. 
entre les deux événements. Dans l'exemple en français, le " donc » permet d'aboutir à la conclusion tout en reformulant la conséquence déjà mentionnée (la locutrice ayant dit : je ne vois pas vraiment un lien..., pour ensuite ajouter : je n'arrive pas à faire un rapport). Malheureusement, le sous-titreur n'est pas parvenu à transférer ces valeurs, il aurait pu choisir une traduction qui soit capable de transmettre cette double fonction de "donc ", en utilisant :

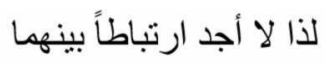

Une telle traduction aurait transmis la conséquence (لذا) et permis une reformulation, au lieu d'opter pour la répétition du même énoncé أرى العال) لألاقة

Les " donc " à orientation récapitulative qui ont été supprimés sont plus nombreux que ceux qui ont été conservés. À titre d'exemple, suite à un reportage sur les pays qui ont connu des révolutions, le présentateur de l'émission dit :

- Exemple 3 : Ben Ali + Moubarak + et donc + Kadhafi

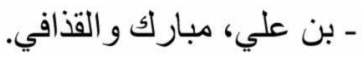

Bien que la traduction soit correcte au niveau sémantique, l'effet produit par la phrase française n'est pas celui produit par la phrase arabe. Et cela est causé par la perte de la valeur discursive du "donc ", marqueur de répétition à orientation récapitulative. Cette valeur aurait pu être rendue par l'usage du lexème arabe أخبر ا (signifiant " enfin "), et la traduction aurait pu être la suivante :

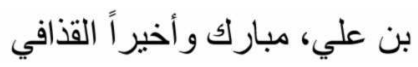

Passons à l'exemple suivant :

- Exemple 4 : non non + justement + pas du tout nous sommes là plus que jamais + puisque + comme vous venez de le dire + nous ne sommes pas du tout satisfaits de cette nouvelle constitution qui a été euh donc voilà + qui a été une constitution vraiment cocotte-minute + nous avions demandé une assemblée constitutionnelle élue par le peuple + ce qui n'a pas été le cas + donc voilà + nos revendications sont encore là

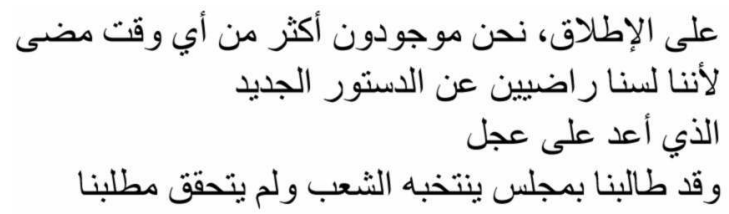

Dans l'exemple ci-dessus, nous pouvons discerner deux occurrences de marqueur complexe (composé d'un marqueur et d'un adverbial), la première occurrence a pour fonction de souligner la progression discursive : elle vient combler une pause qui permet à la locutrice de trouver le mot approprié, c'est la raison pour laquelle sa disparition n'entraîne aucun effet négatif sur l'énoncé sous-titré. 
La deuxième occurrence a pour fonction de reformuler et d'expliciter. Nous pouvons distinguer un énoncé-source (nous sommes là plus que jamais), un énoncé-doublon (nos revendications sont encore là) et un élément qui transmet la relation paraphrastique, à savoir "donc ".

Or, cette valeur paraphrastique aurait pu être maintenue au lieu d'être supprimée. Notre suggestion est ici d'ajouter un nouveau sous-titre et de traduire la dernière phrase tout en ajoutant la particule أي capable de transférer la valeur de la reformulation :

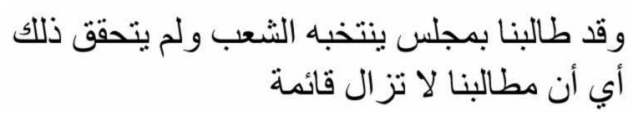

En revanche, la suppression du " donc " de transition participative n'a eu aucun effet négatif sur le sous-titrage, et pour cause : le changement de plan et le passage de la caméra de l'interviewée au présentateur (et vice-versa) suffisent à souligner cette transition. Comme tout lecteur arabophone peut le remarquer, la traduction cible n'a pas été affectée par la disparition du " donc".

L'exemple suivant nous permet d'éclaircir le " donc » marqueur de structuration conceptuelle :

- Exemple 5 : bienvenue à tous place Tahrir + place Tahrir l'un des symboles du printemps arabe + il y a donc dix ans + jour pour jour + les attentats du 11 septembre bouleversaient la planète

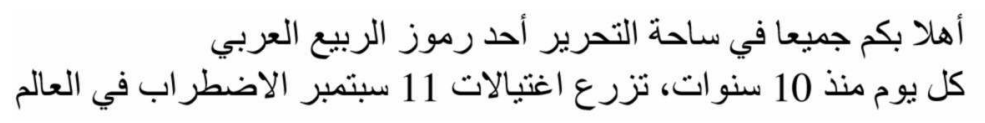

Dans l'énoncé précédent, " donc " permet au présentateur de passer du mot d'accueil adressé aux téléspectateurs au thème principal de l'émission, à savoir la relation entre le 11 septembre et le printemps arabe. Dans ce cas, "donc " n'est pas seulement et simplement un marqueur de structuration conceptuelle mais aussi un marqueur de prosodie qui a pour fonction de segmenter l'information et de ponctuer le discours. Malheureusement, rien de tel ne transparait dans le sous-titrage arabe. Si le passage au thème principal se fait facilement en français grâce à " donc ", l'énoncé arabe paraît saccadé.

La dernière fonction de " donc " qui mérite d'être mentionnée, est celle de ponctuant qui permet d'attirer l'attention sur les éléments constitutifs du discours. C'est le cas par exemple de l'énoncé suivant:

- Exemple 6 : deux invités nous ont quittés + nous remercions donc Beithi Lachqar + nous remercions aussi Hala Galal qui étaient donc sur ce plateau

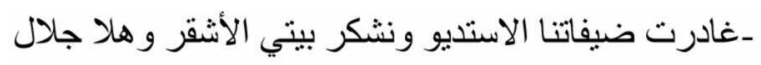


Si dans l'énoncé français, "donc " reçoit une intonation montante, cette nuance ne transparaît évidemment pas dans le sous-titrage, et c'est là un des problèmes majeurs du passage de l'oral à l'écrit.

Notons également que " donc " a joui dans l'ensemble de notre corpus d'une véritable liberté distributionnelle : si le connecteur a toujours occupé une position initiale par rapport à l'unité discursive dans laquelle il se trouvait, le marqueur a été le plus souvent interposé par rapport à cette unité et parfois même postposé. L'étude de "donc » nous mène à établir le parallélisme suivant entre la V0 et la VST :

\begin{tabular}{|l|c|c|}
\hline \multicolumn{1}{|c|}{ Valeur sémantique et discursive } & Version originale & Version sous-titrée \\
\hline & Activée $(+/-)$ & Activée $(+/-)$ \\
\hline Conséquence & + & + \\
\hline Récapitulation & + & + \\
\hline Reformulation & + & - \\
\hline Transition participative et conceptuelle & + & - \\
\hline Ponctuant & + & - \\
\hline
\end{tabular}

Tableau 5. Comparaison entre les valeurs sémantiques et discursives activées dans les deux versions

\section{2. "Alors " et les tours de parole}

Le deuxième MD que nous étudierons est " alors ". II a trois valeurs : une valeur temporelle exclusive, une valeur argumentative exclusive ou les deux à la fois. De façon générale, "alors " permet de relier les énonciations, de créer la cohésion et par là, d'ancrer le discours dans la situation d'énonciation. C'est un indicateur d'ancrage interpropositionnel.

"Alors ", de par sa fonction anaphorique, rappelle une situation connue des interlocuteurs, ce qui en fait un garant de la continuité thématique.

Selon Gerecht (1987), " alors » a trois emplois : opérateur temporel (il peut souligner la concomitance pleine, la concomitance partielle, la successivité et l'antériorité), connecteur argumentatif (lorsqu'il présente l'énoncé p comme la cause de l'énoncé q) et marqueur du discours.

"Alors ", qui figure 15 fois dans notre corpus, a été maintenu 9 fois dans le sous-titrage et supprimé 6 fois, ce qui signifie que presque $60 \%$ des occurrences ont été transmises dans la version sous-titrée. 


\begin{tabular}{|l|l|c|c|}
\hline \multicolumn{2}{|l|}{} & Fréquence & Pourcentage \\
\hline \multirow{2}{*}{ Type de lien } & Conséquence-conclusion & 6 & $40 \%$ \\
& Temporel & 1 & $6,6 \%$ \\
& Lien entre deux énonciations & 4 & $26,6 \%$ \\
& Renvoi à une situation antérieure & 1 & $6,6 \%$ \\
& Lien entre un savoir commun et une énoncia- & 3 & $20 \%$ \\
\hline & tion explicitée & 15 & $100 \%$ \\
\hline
\end{tabular}

Tableau 6. Différentes fonctions de " alors » dans la VO

\begin{tabular}{|l|l|c|c|}
\hline \multicolumn{1}{|l|}{} & Maintien & Disparition \\
\hline \multirow{2}{*}{ Type de lien } & Conséquence & 6 & 0 \\
& Temporel & 1 & 0 \\
& Lien entre deux énonciations & 1 & 3 \\
& Renvoi à une situation antérieure & 0 & 1 \\
& Lien entre un savoir commun et une énoncia- & 1 & 2 \\
\hline & tion explicitée & 9 & 6 \\
\hline \multicolumn{1}{|c|}{ Total } & 9 \\
\hline
\end{tabular}

Tableau 7. Nombre d'occurrences d'» alors " maintenues ou supprimées dans la VST

Ce marqueur a été conservé lorsqu'il avait une fonction conclusive qui s'appuyait sur un savoir commun à tous les interlocuteurs. Dans ce cas, les contreparties arabes du marqueur étaient : إذاً، بالتالي ولذا :

- Exemple 7 : je faisais partie du groupe qui n'en voulait pas de ce système et qui voulait changer + mais eux + ils ne voulaient pas ce système + alors c'est nous qu'ils ont changés + alors une fois que j'ai réussi en 2010 [...] le lendemain mon nom avait disparu

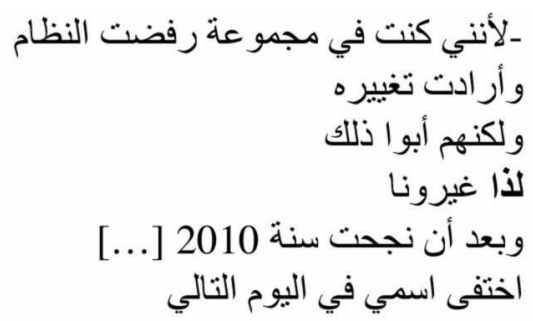

Dans cet énoncé, nous remarquons la présence de deux occurrences du marqueur "alors ", le premier, consécutif, introduit une suite au discours, tandis que le second marque la 
progression discursive (il aide la locutrice à segmenter son énoncé en unités informatives, facilitant ainsi l'interprétation par le destinataire). Toutefois, seul le premier " alors " a été maintenu dans le sous-titrage arabe.

"Alors " ayant comme fonction de relier deux énonciations explicitées a le plus souvent été supprimé :

- Exemple 8 : L1 : c'est-à-dire les États-Unis + Israël dictaient notre politique extérieure.

$\mathrm{L} 2$ : alors + ça c'est pour le domaine de la politique étrangère + mais vous + vous avez été parlementaire

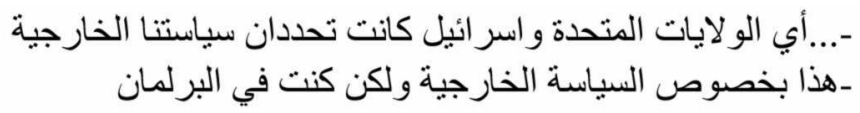

Il en est de même lorsque " alors " vient en début d'énoncé et a pour fonction d'attirer l'attention de l'interlocuteur et de s'assurer de sa participation à la conversation.

Dans les cas où " alors " a un rôle injonctif permettant d'introduire un nouvel interlocuteur et de l'inviter à s'exprimer, le sous-titreur a également opté pour la suppression du marqueur.

Dans l'exemple suivant, le présentateur de l'émission, tout en évoquant le premier Aïd Al-Fitr (petit Baïram) de l'après Ben Ali, demande à l'invitée si le retour de l'esprit religieux en Tunisie l'inquiète :

- Exemple 9 : non + ça ne me dérange pas parce que + j'ai vécu toute ma vie en Tunisie ++ je me suis habituée [...] alors ++ c'est vrai que le jour de l'Aïd était vraiment spécial en Tunisie

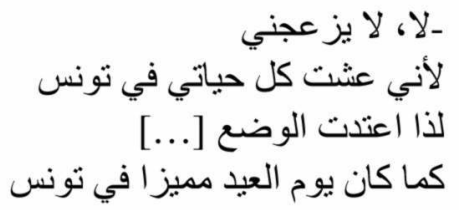

La valeur discursive du marqueur permet ici à la locutrice de reprendre le thème principal de la question après avoir donné son avis concernant le retour de la religion sur la scène sociale. Dans ce cas, l'énonciation de " alors " vient boucler une séquence discursive et délimiter une nouvelle séquence thématique. C'est comme si " alors " permettait la reprise du thème principal suite à une incise ou interruption. Dans ce cas, il a un fonctionnement dialogique (intrainterventions). II aide la locutrice à conserver la parole et sert d'appui pour la suite de l'énoncé.

Rien de tel n'apparaît dans le sous-titrage, d'autant plus que le sous-titreur a omis " c'est vrai " de sa traduction. Néanmoins la solution que nous proposons est d'avoir recours à la particule d'affirmation نعe (oui) qui permet de transmettre la véracité de son énoncé, voire sa modalité assertive : 


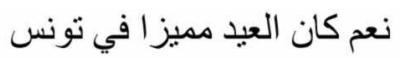

Finalement, quand le marqueur " alors " a pour rôle de relier un savoir commun (le rôle des femmes dans les révolutions arabes) à une nouvelle énonciation (présentation d'une cuisinière égyptienne), c'est-à-dire lorsque son fonctionnement est dialogal (inter-interventions), le sous-titreur le remplace par إليكم, qui signifie " voici » :

- Exemple 10 : alors + une belle histoire maintenant + qui est née de ce printemps arabe

$$
\text { إلبكم قصة جديدة ولات من الربيع العربي }
$$

II nous est possible de conclure que " alors " à valeur conclusive ou consécutive a été conservé, tandis que le marqueur discursif " alors "à valeur injonctive (permettant de lier deux énonciations ou une situation de communication et une énonciation ou encore un savoir commun et une énonciation) a été supprimé.

\section{3. " Là " et " voilà "}

En principe, "là " est un déictique dont la référence est liée à la situation d'énonciation. Sa valeur peut être soit locative, soit temporelle. Les linguistes s'accordent à voir l'identification référentielle, dans laquelle la particule se joint à un groupe nominal pour souligner la référence, comme un emploi principal de "là ". Et bien que cette identification toujours liée aux adjectifs démonstratifs ait un équivalent en arabe (أسماء الإشتارة), le sous-titreur a opté dans tous les cas pour l'omission de cette référentialité.

Lorsque la référence de la particule était locative, deux options se sont présentées au soustitreur : l'omission ou l'explicitation.

- Exemple 11 : donc + là + bon aujourd'hui c'est calme

$$
\text { الهدوء سائد اليوم }
$$

Dans cet exemple, la locutrice fait référence à l'endroit où elle se trouve, à savoir le siège du ministère des Affaires étrangères. Or, le sous-titrage efface cette valeur, bien qu'il existe une équivalence en arabe, à savoir هناك qui est l'antonyme d'» ici ", traduit par هن . Toutefois, cette suppression n'entraîne pas d'effet négatif sur la compréhension par le téléspectateur, puisque l'image vient transférer la valeur locutive et qu'ils sont en mesure de voir l'endroit désigné par la locutrice.

Dans l'exemple suivant, le sous-titreur a choisi l'explicitation de la référence de la particule, à savoir le studio de télévision. Une telle explicitation était superflue, puisque la scène se déroule dans un studio et que le sous-titre est, de ce fait, devenu très long. 
- Exemple 12 : avant la révolution + ça aurait été impossible d'être là

$$
\text { قبل الثورة استحال على المجيء إلى استديو تليفزيوني كهذا }
$$

La référence temporelle, quant à elle, est parfois établie dans l'énoncé par un renvoi anaphorique. Dans l'exemple suivant, elle ne désigne pas le moment d'énonciation mais renvoie plutôt à dix ans auparavant.

- Exemple 13 : il y a donc dix ans + jour pour jour + les attentats du 11 septembre bouleversaient la planète + et pour de nombreux dirigeants en Occident + eh bien + c'était Moubarak et Ben Ali contre Ben Laden + et là + il valait mieux une dictature plutôt que le terrorisme

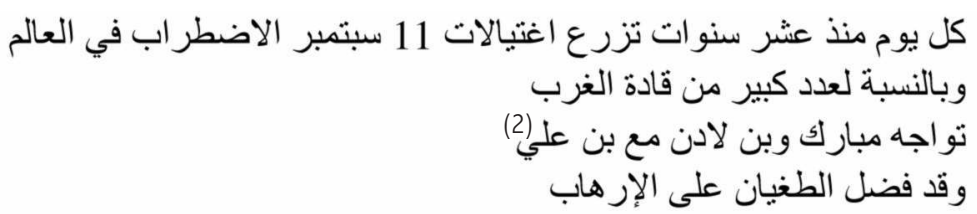

Dans cet énoncé, " là " aurait pu être remplacé par "à l'époque ". On regrette que le soustitreur n'ait pas conservé cette valeur discursive en écrivant :

$$
\text { و آنذاك، تم تفضيل الديكتاتورية على الإرهاب }
$$

Par contre dans l'exemple ci-dessous, "là " peut être remplacé par " à ce moment-là " et sa référence est postérieure au moment de l'énonciation. "Là " est venu renforcer la valeur de " quand".

- Exemple $14:$... et je pense que + quand les gouvernements + ou les politiques lâcheront cette politique ++ de la peur + pour gouverner + je pense que là + on ira vers une autre vision des effets négatifs

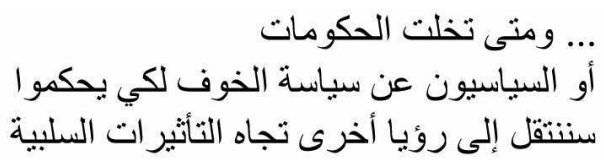

La traduction que nous proposons est "par la suite " (حينـذ) qui aurait pu être antéposé au verbe سننتقل (on ira).

La particule a parfois une fonction cataphorique, c'est-à-dire qu'elle annonce un nouveau segment linguistique.

(2) Notons que le sous-titreur a commis un contre-sens puisqu'il aurait dû écrire :

وقد تواجه مبارك وبن علي مع بن لادن 
- Exemple 15 : oui + il a raison mais aussi + je pense que + nous avons parti tous dans la rue + là + ça c'est un mouvement international

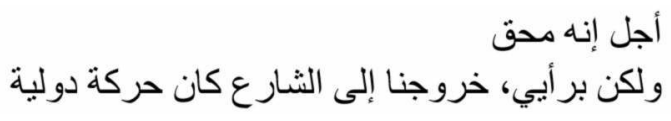

À cet égard, le rôle argumentatif de la particule est à remarquer. II permet de poser des prémisses et de garantir la progression thématique.

Le locuteur commence par poser des prémisses ou encore reprendre une partie du raisonnement antérieur pour ensuite asserter, résoudre le problème. Cette mise en relation explique l'occurrence fréquente du là entre deux propositions: il ne sert pas, à la façon des connecteurs, à effectuer l'enchaînement mais plutôt à établir au gré de l'énonciation du message, sa pertinence argumentative. (Forget, 1989 : 77)

Ce faisant, " là " aurait pu être traduit par حقا (littéralement " vraiment ") :

$$
\text { -ولكن برأيب، خروجنا إلى الثار ع كان حقاً حركة دولية }
$$

Idem pour l'exemple suivant, le locuteur a recours à la particule " là " pour délimiter des unités de discours et pour signaler leur importance au destinataire, selon leur contribution au plan contextuel :

- Exemple 16 : je pense qu'on a passé ++ déjà on a passé à autre chose + c'est pas maintenant + dix ans après + qu'on a passé à autre chose + là + je suis entièrement d'accord avec ce que Rim vient de dire

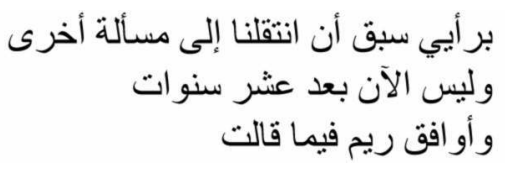

Raison pour laquelle, nous proposons de traduire " là " par في هذا الثأن (à cet égard).

En guise de conclusion, nous pouvons indiquer que dans la version originale la particule apparaît à différents endroits de la chaîne parlée, conformément à la segmentation informationnelle à laquelle procède le locuteur. Malheureusement, le sous-titrage a effacé toutes ces pertinences, laissant un énoncé non pas vide de sens, mais vide de pertinence et d'effet énonciatif. 


\begin{tabular}{|l|c|c|}
\hline \multicolumn{1}{|c|}{ Valeurs de " là " } & Version originale & Version sous-titrée \\
\hline & Activée (+/-) & Activée (+/-) \\
\hline Valeur référentielle & + & - \\
\hline Valeur locative & + & + \\
\hline Valeur temporelle & + & - \\
\hline Fonction cataphorique & + & - \\
\hline Fonction argumentative & + & - \\
\hline Délimitation des unités discursives & + & - \\
\hline
\end{tabular}

Tableau 8. Valeurs de " là " dans les deux corpus

Passons maintenant à " voilà ". Indicateur de la structure de l'interaction, "voilà " est un appui discursif. II peut paraître intégré dans la séquence sur laquelle il porte ou en être détaché, surtout en initiale. En principe, cette particule a plusieurs fonctions, qui sont : la confirmation, l'acquiescement, la clôture de tâche, la présentation et la fonction phatique. "Voilà " paraît dans notre corpus soit seul, soit en combinaison avec d'autres conjonctions de type " puis " ou "donc".

- Exemple 17 : euh + euh + ce retour de fierté ++ on va finalement peut-être reconnaître ce que c'est un arabe + ou ce que c'est un berbère + ou ce que c'est un africain ou ++ et puis voilà + un retour des identités ++ une fierté des identités oui + mais pas seulement arabe

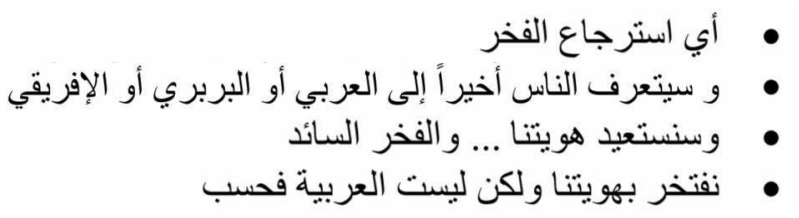

Dans l'exemple précédent, " voilà " aide la locutrice à structurer son intervention, et à confirmer son idée à la fois sur l'axe paradigmatique et l'axe syntagmatique.

-....un retour de fierté $\{a+b\}$

- ....un retour des identités $\{a+c\}$

- ....une fierté des identités $\{b+c\}$

Dans ce cas, " voilà " a une fonction récapitulative. En ce sens, nous proposons la traduction suivante, qui respecte la coordination entre les différents groupes nominaux en arabe :

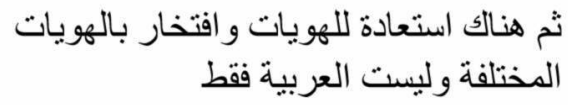


Dans l'exemple suivant, " voilà » introduit un effet de surprise et de contradiction : les demandes des révolutionnaires n'ont pas été satisfaites, contre toute attente.

- Exemple 18: nous demandons à ce qu'il il il règne + sans gouverner + comme ce qui se passe en Espagne + et puis + surtout voilà avec la nouvelle constitution + il a gardé tous ses pouvoirs

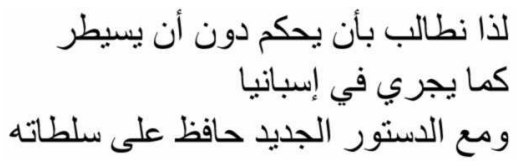

Nous proposons que le sous-titre transfère ce double effet d'opposition et de présentation en ayant recours à la structure ولكن ها هو (littéralement : " mais le voilà ") :

$$
\text { ولكن ها هو يبقي على سلطاته في الاستور الجديد }
$$

Toutes les occurrences de "voilà " ont été effacées dans la version sous-titrée et de fait désactivées.

\subsection{Les marqueurs discursifs propositionnels}

Les MD propositionnels (MDP) sont constitués d'un groupe de marqueurs discursifs qui contiennent un verbe conjugué, ce qui les assimile à des propositions (en termes morpho-syntaxiques), bien qu'ils soient considérés comme des marqueurs discursifs puisqu'ils sont figés dans des formes invariables.

Les fonctions les plus importantes des MDP sont:

- une fonction de structuration du discours aux niveaux syntaxique, thématique et discursif, ce qui implique une forte désémantisation par rapport à la signification du départ ;

- une fonction phatique qui peut être la recherche d'approbation discursive, la marque de politesse ou de camaraderie ;

- une fonction d'instrument de prise de tour. (Andersen, 2007 : 26)

\subsubsection{Les MDP avec le verbe "savoir "}

Le verbe " savoir " apparaît dans certains MDP, soit à la première personne du singulier, soit avec le pronom indéfini " on ". Ce qui est remarquable, c'est que dans toutes les occurrences le verbe est à la forme négative.

Avec la première personne du singulier, les MDP constituent un point d'ancrage véhiculant les attitudes et les commentaires du locuteur. Ils aident l'interlocuteur à mieux interpréter le discours mais aussi à se positionner par rapport à ce discours. 
Dans les cas de la personne " je ", le sous-titreur a transmis le MDP une fois et l'a omis une autre fois.

- Exemple 19 : on n'a pas vraiment + beaucoup entendu le mot de terroriste + ils ont dit extrémiste + ou + je ne sais pas quoi

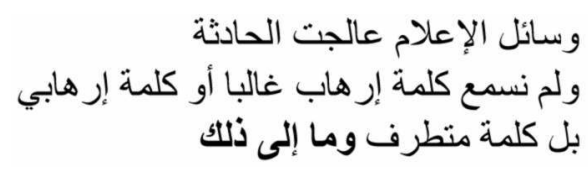

Par le recours au MDP « je ne sais pas quoi ", le locuteur affiche son hésitation, son manque d'assurance et implique l'interlocuteur dans le traitement de l'énoncé. En ce sens, la traduction arabe est réussie.

L'emploi du pronom indéfini " on " ne contribue pas vraiment à la progression discursive mais définit plutôt le cadre relationnel entre le locuteur et son interlocuteur. Sa fonction est ainsi interactionnelle. II anticipe les objections ou les corrections de l'interlocuteur. C'est la raison pour laquelle le sous-titreur l'a traduit.

- Exemple 20 : rapidement + où en est le mouvement du 20 février + est-ce qu'il existe ou + est-ce que ++ on ne sait pas trop + on a l'impression finalement qu'avec les annonces + les annonces de ces réformes + le mouvement est borné

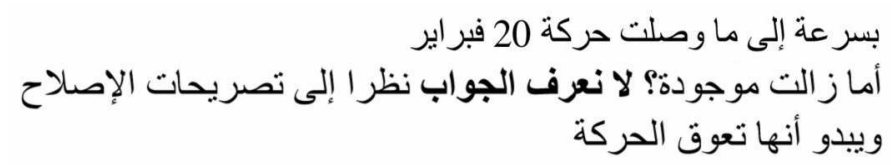

\subsubsection{Les MDP avec le verbe "dire"}

Le verbe " dire " introduit une nuance, une assertion qui paraît peu discutable, une conclusion logique, une délimitation d'un cadre de validation argumentative et d'exemplification, une feinte acceptation, une orientation de la discussion sur un point particulier, une proposition.

Le verbe " dire " figure dans cinq MDP, trois fois à la première personne (je) et deux fois à l'infinitif.

- Exemple 21 : mais + en même temps + vous ne le voyez pas mais + c'est quand même ++ il y a quand même eu des effets négatifs + ça vous ne le niez pas je veux dire + il était beaucoup plus difficile par exemple quand on est arabe musulman de voyager

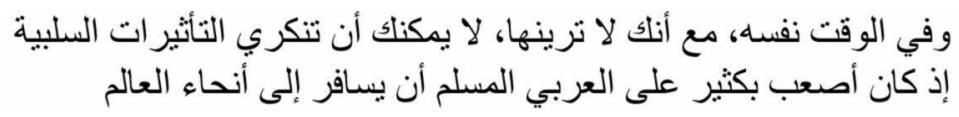


Dans l'exemple précédent, le MDP a pour rôle de souligner le degré d'engagement du locuteur dans son acte. Un engagement effacé dans la version sous-titrée, alors que le traducteur aurait pu avoir recours aux verbes arabes أقصد ou أعني

Si avec les MDP " je veux dire ", le sous-titreur a opté pour la suppression, dans les cas où le verbe " dire " était à l'infinitif, il l'a maintenu.

- Exemple 22 : c'est le + le + le ++ comment dire ++ l'autre extrême un peu

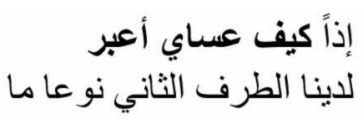

Le " comment dire " accompagne une reformulation et indique que le locuteur est en train de chercher le ou les mots appropriés. II constitue une sorte de protection contre le côté négatif de ce qui est dit par un appel à l'interlocuteur d'exprimer son acceptation.

\subsubsection{Les MDP avec le verbe "voir"}

Le verbe " voir " paraît dans le MDP " vous voyez " qui marque la fin d'une unité discursive.

- Exemple 23 : donc on les tapait au niveau des pieds [...] pour qu'ils n'aillent plus à la mosquée + vous voyez ++ c'est donc ++ c'est un autre extrême aussi et + ce n'est pas normal

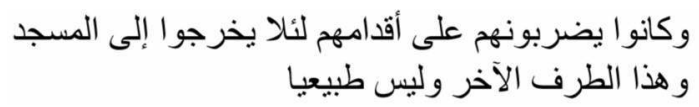

Le MDP " vous voyez " a en principe un caractère explicatif par rapport au contexte. Dans ce cas, le verbe "voir " n'a pas son sens premier de " perception par les yeux ", mais plutôt le sens de la compréhension. II permet, en quelque sorte au locuteur de s'assurer que son destinataire le suit dans son raisonnement. On regrette que le sous-titreur ait omis ce marqueur phatique qui aurait pu facilement être traduit par أتفهمني

$\|$ en résulte un énoncé moins prudent, moins connivant que dans la version originale, ce qui modifie la nature de la relation entre le locuteur et son destinataire.

En effet, par l'utilisation de verbes parenthétiques (en incise), la construction du sens se fait sans mettre en péril la crédibilité de l'autre. Or, rien de ceci n'apparaît dans le sous-titrage. L'effet sémantique transmis par les MDP n'est pas véritablement assuré.

\section{Conclusion}

Le sous-titrage est un processus sélectif dans lequel le traducteur opte pour la condensation, la reconstitution et la paraphrase. À la question que nous nous sommes posée au début de 
l'article, "le sous-titrage mène-t-il à une sous-information ? ", la réponse semble être " pas de manière excessive ". Y a-t-il un sous-effet et un manque de pertinence dans la réception ? Certes. Notre étude prouve que dans les exemples choisis les marqueurs discursifs jouent un rôle moindre au niveau " vertical " de la communication (entre l'émission et les récepteurs) qu'au niveau " horizontal " (entre le présentateur du programme télévisé et ses invités).

Notre étude s'interrogeait sur l'interprétation fonctionnelle de certaines formes et configurations verbales, à savoir les marqueurs discursifs. Ceux-ci constituent des marques explicites de l'articulation discursive et transmettent, en français, les mouvements de construction de la référence et la gestion de l'intersubjectivité.

L'analyse contrastive et qualitative des MD entre le français et l'arabe nous a permis de mettre en lumière des points de divergence et de convergence interlinguistiques ainsi que les fonctions intralinguistiques des MD (en français).

Ainsi :

II semble ainsi possible de dire que la présence des MD est liée à deux facteurs principaux. En effet, d'une part "la progression discursive " - qui correspond à la planification et à l'emploi référentiel - et d'autre part "l'interaction " - qui correspond à la politesse et à l'emploi du type interpersonnel - expliqueront la présence des marqueurs discursifs dans l'échange oral. (Denturck, 2007-2008 : 22)

Concernant la version sous-titrée, nous avons remarqué la présence de trois cas de transmission de la valeur sémantique des MD : le sens a été rendu, le sens a été approximativement rendu, le sens a été occulté. En fonction de ces cas, la distance entre les langues française et arabe a été soit agrandie soit amoindrie dans le sous-titrage.

Notre étude montre qu'il n'y a pas de correspondance simple d'un MD de la langue source en langue cible. Alors que les MD consécutifs et conclusifs ne posent pas de problèmes majeurs pour la traduction du fait de la relative simplicité de leur valeur sémantique, les particules posent davantage de problèmes, soit parce qu'elles n'ont pas d'équivalent en arabe, soit parce que le sous-titreur n'a pas tenu compte de leur valeur.

Si en sémantique discursive le contenu propositionnel d'une séquence importe moins que sa formulation, la majeure partie de la valeur des MD a été occultée dans le sous-titrage, ce qui en a, en quelque sorte, limité la pertinence. Malheureusement, pour les sous-titreurs, les MD sont des éléments périphériques dans les énoncés qui composent la dynamique de l'échange. En somme, cette étude nous a permis de rejoindre Mason (1989), Hatim et Mason (1997) et Kovacic (1998) selon lesquels les marqueurs, d'hésitation surtout, sont systématiquement perdus lorsqu'ils sont transférés en mode écrit. Nous ajoutons que plus de la moitié des marqueurs discursifs n'ont pas été transférés. Si le contenu propositionnel n'en est pas affecté, la pertinence de l'énoncé et la perception qu'en ont les téléspectateurs sont lésés. 
Saussure a été le premier à souligner que le signe était la combinaison d'un signifié et d'un signifiant. Nous aimerions, quant à nous, y ajouter une troisième composante, celle de l'effet significatif, qui joue un rôle important dans l'élaboration du sens.

ranoadel@gmail.com

Rania A. Khalifa est professeure adjointe de linguistique française à la faculté des Langues (Al-Alsun) de l'Université d'Ain-Chams en Égypte. Titulaire d'un master et d'une thèse de doctorat en sociolinguistique, elle a ensuite préféré orienter ses recherches vers différents champs de la linguistique : analyse de discours, argumentation, traductologie, etc. Elle a publié dans des revues américaines, canadiennes, françaises, roumaines, égyptiennes et saoudiennes.

\section{Bibliographie}

\section{Livres et articles}

- Adam, J. M., (1990), Éléments de linguistique textuelle, Bruxelles-Liège (Belgique), Mardaga.

- Andersen, H. L., "Marqueurs discursifs propositionnels " in Langue française, 2007/2 $n^{\circ}$ 154, Armand Colin, p.13-28.

- Auchlin, A. (1981), "Réflexions sur les marqueurs de structuration de la conversation " in Études de linguistique appliquée, 44, p. 88-103.

- Blanche-Benveniste, C., (1997), Approches de la langue parlée en français, Paris, Ophrys.

- Bolly, C. \& Degand L., (2009), "Quelle(s) fonction(s) pour donc en français oral ? Du connecteur conséquentiel au marqueur de structuration du discours " in Lingvisticae Investigationes, numéro 32, p. 1-32.

- Dostie, Pusch D., Claus, (2007) "Présentation des marqueurs discursifs, sens et variation", in Langue française, $n^{\circ}$ 154, 2007-2, Armand Colin, p. 3-12.

- Dostie, G., (2004), Pragmaticalisation et marqueurs discursifs, analyse sémantique et traitement lexicographique, Belgique, Duculot.

- Ducrot, O., (1980), Les mots du discours, Paris, Minuit.

- Ducrot, O. et Anscombre, J.-C., (1983), L'argumentation dans la langue, Bruxelles, Mardaga.

- Fernandez MMJ, (1994), Les particules énonciatives, Paris, PUF. 
- Forget, D., "Là : un marqueur de pertinence discursive ", in Revue québécoise de linguistique, vol. $18, n^{\circ} 1,1989$, p. 57-82.

- Fraser, B., (1999), "What are discourse markers ? ", in Journal of Pragmatics, 31, Elsevier, p. $931-952$.

- Gerecht, M.-J., (1987) «Alors, opérateur temporel, connecteur argumentatif et marqueur de discours ", in Cahiers de linguistique française $8+1987$, Nouvelles approches des connecteurs argumentatifs, temporels et reformulatifs, Université de Genève, p. 69-79.

- Gulich, E et Kotschi, T. (1983), "Les marqueurs de reformulation paraphrastique " in Cahiers de linguistique française 5, p. 305-346.

- Hansen, M.-B. (1997) "Alors and donc in spoken french : a reanalysis " in Journal of pragmatics, 28(2), p. 153-187.

- Hatim, B. et Mason, I. (1997), "Politeness in screen translation ", in L. Venuti (ed.) The Translation Studies Reader (2000), Londres et New York, Routledge, p. 430-445.

- Kovacic, I., (1998), "Six subtitlers, six subtitling texts ", in L. Bowker, M. Cronin, D. Kenny, J. Pearson (ed.), Unity in Diversity? Current Trends in Translation Studies, Manchester, St Jerome, p. 75-82.

- Mason I., (1989), "Speaker meaning and reader meaning: preserving coherence in screen translating ", in R. Kölmer, J. Payne (ed.), Babel. The cultural and linguistic barriers between nations, 1989, Aberdeen University Press, Aberdeen (Écosse), p. 13-24.

- Marleau, L., "Les sous-titres, un mal nécessaire ", in Méta : Journal des traducteurs, volume 27, numéro 3, septembre 1982, Les Presses de l'Université de Montréal (Canada), p. $271-285$.

- Schiffrin, D. (1987), Discourse Markers, Cambridge (Angleterre) : C.U.P.

- Zenone, A. (1981), "Marqueurs de consécution : le cas de donc " in Cahiers de linguistique française 2, p. 113-139.

\section{Thèses et études académiques}

- Denturck, E., Étude des marqueurs discursifs, l'exemple de quoi, Faculteit Taal-en Letterkunde Sectie 2 talen Academiejaar 2007-2008, Université de Gand (Belgique). 